\title{
Baryon decuplet in the chiral dynamics of $\Lambda$ hyperons in nuclear matter
}

\author{
J. Martín Cámalich and M. J. Vicente Vacas \\ Departamento de Física Teórica and IFIC, Centro Mixto Universidad de Valencia CSIC, \\ Institutos de Investigación de Paterna, Aptdo. 22085, E-46071 Valencia, Spain
}

(Received 27 November 2006; published 28 March 2007)

\begin{abstract}
We study the long range part of the $\Lambda$-hyperon optical potential in nuclei using quantum many body techniques and flavor-SU(3) chiral Lagrangians as starting point. More precisely, we study the contributions to the $\Lambda$-hyperon optical potential due to the long-range two-pion exchange, with $\Sigma$ and $\Sigma^{*}$ baryons in the internal baryonic lines and considering $N h$ and $\Delta h$ excitations. We also consider the contribution to the spin-orbit potentials that comes out from these terms. Our results support a natural explanation of the smallness of the $\Lambda$-nuclear spin-orbit interaction and show the importance of the $\Sigma^{*}$ and $\Delta$ degrees of freedom for the hyperon-nucleus interactions.
\end{abstract}

DOI: 10.1103/PhysRevC.75.035207

PACS number(s): 21.80.+a, 21.65.+f, 13.75.Ev, 24.10.Cn

\section{INTRODUCTION AND FRAMEWORK}

The interaction of $\Lambda$ hyperons $(Y)$ with nucleons $(N)$ and nuclei has been the subject of much work during the last decades [1-8]. One of the main goals in the field is to relate the hypernuclear observables to the bare $Y N$ interaction, i.e., in Refs. [9-11]. Although there are potentials that describe very well the $Y N$ scattering phenomenology [12-14] there is still considerable freedom due to the scarcity of available $Y N$ data, and the analysis of hypernuclear observables could add further constraints to the potentials.

One of the interesting features of the $\Lambda$ nucleus potential is the weakness of the spin-orbit interaction. After some phenomenological analysis [15], and the calculations of Brockmann and Weise [16] it was experimentally confirmed [17] that the $\Lambda$ nucleus spin-orbit interaction was at least one order of magnitude smaller than for the nucleon-nucleus case. (See also [18-20] for other experiments supporting this result.) Several theoretical approaches have tried to explain it, ranging from one boson exchange (OBE) potentials [2,10,21,22] with the couplings sometimes motivated by the underlying quark dynamics, to the consideration of two meson exchange pieces [16] or to quark based models [23-25].

Recently, the $\Lambda$ [26] and $\Sigma$ [27] hyperons mean field and spin-orbit interaction have been studied using an effective field theory approach, which already has been successful in the description of binding and single particle properties of nucleons in nuclear matter [28-30]. Starting with the leading order chiral meson baryon octet Lagrangian the long range contributions to the potential coming from one kaon and two pion exchange were evaluated, finding among other results a natural explanation of the spin-orbit weakness due to a cancellation of short and long range pieces.

The main contribution in Ref. [26] to the $\Lambda$ mean field comes from diagram (a) of Fig. 1. This term is related to the pion self-energy coming from a nucleon-hole excitation in nuclear matter. On the other hand, it is well known from pion physics the relevance of $\Delta$-hole excitations for the pion selfenergy even at very low energies well below the $\Delta$ peak [31]. The large coupling $\pi N \Delta$ is responsible for this. Furthermore, in purely nucleonic matter it has been found that the real singleparticle potential is substantially improved by the inclusion of the $\pi N \Delta$-dynamics [30]. Also, in Refs. [12,32] the importance of the decuplet baryons as intermediate states in the two meson exchange terms of the $Y N$ bare potential was shown.

Our aim in this paper is to extend the work of Ref. [26] considering also the interaction with the relevant baryons of the decuplet ( $\Delta$ and $\Sigma^{*}$ ) and its contribution to the two pion exchange potential. In particular, we will study whether the natural explanation of weakness of the spin-orbit $\Lambda$-nucleus potential is still valid after the inclusion of the new terms.

The coupling between the pseudoscalar meson octet and the baryon octet is given by the lowest order SU(3) chiral meson baryon Lagrangian

$$
\mathcal{L}_{\text {oct }}=D \operatorname{Tr}\left(\bar{B} \gamma_{\mu} \gamma_{5}\left\{u^{\mu}, B\right\}\right)+F \operatorname{Tr}\left(\bar{B} \gamma_{\mu} \gamma_{5}\left[u^{\mu}, B\right]\right),
$$

where $B$ is the traceless flavor matrix accounting for the spinor fields of the baryons octet $(N, \Lambda, \Sigma, \Xi)$, and $u^{\mu}=$ $i\left[\xi^{\dagger}, \partial^{\mu} \xi\right] / 2, \xi=\operatorname{Exp}\left(i \Phi / \sqrt{2} f_{\pi}\right)$ introduces the $\mathrm{SU}(3)$ matrix of meson fields $\Phi$. The $B$ and $\Phi$ matrices are normalized as in [33]. The parameter $f_{\pi}=92.4 \mathrm{MeV}$ is the weak pion decay constant and $D=0.84, F=0.46$ are the $\mathrm{SU}(3)$ axial-vector coupling constants for the octet baryons. These values of $D$ and $F$ have been taken to facilitate the comparison with [26]. As discussed in [26], they lead to $K N \Lambda, \pi \Lambda \Sigma$, and $\pi N N$ coupling constants consistent with their empirical values [34].

The interaction between the baryon octet, the baryon decuplet, and the meson octet is described by [35]

$$
\mathcal{L}_{\mathrm{dec}}=\frac{\mathcal{C}}{\sqrt{2} f_{\pi}}\left(\sum_{a, b, c, d, e} \epsilon_{a b c} \bar{T}_{\mu}^{a d e} \partial^{\mu} \Phi_{d}^{b} B_{e}^{c}+\text { h.c. }\right),
$$

being $\mathrm{T}_{a b c}^{\mu}$ the $\mathrm{SU}(3)$ representation for the $3 / 2^{+}$decuplet fields [35] and where we have expanded the axial current up to one meson field. The analysis of the partial decay widths of the decuplet shows a breaking of the $\mathrm{SU}(3)$ symmetry $[35,36]$ of the order of $30 \%$. In our calculation we need the $\Sigma^{*} \pi \Lambda$ and $\Delta \pi N$ vertices and we will use for each case as coupling constant $\mathcal{C}$ the value fitted to the decay widths of $\Sigma^{*} \rightarrow \pi \Lambda$ and $\Delta \rightarrow \pi N$, respectively $\left(\mathcal{C}_{\Sigma *}=1.7, \mathcal{C}_{\Delta}=2.0\right)$. 


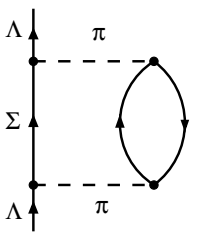

(a)

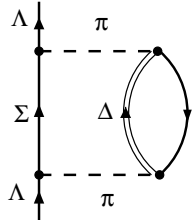

(b) (c)

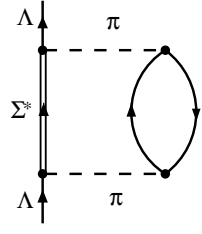

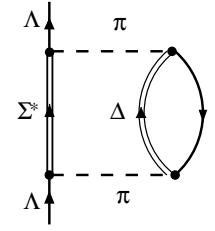

(d)
FIG. 1. Two pion exchange diagrams with $\Sigma, \Sigma^{*}$ in intermediate states and with $1 N h$ and $1 \Delta h$ excitations.

\section{II. $\Lambda$-NUCLEUS CENTRAL POTENTIAL}

We focus on the density dependence of the mean-field $U_{\Lambda}\left(k_{f}\right)$ for a zero-momentum $\Lambda$-hyperon interacting with isospin-symmetric nuclear matter. The depth of this potential at the saturation nuclear density $\rho_{0}$ is around $-30 \mathrm{MeV}$. The only one meson exchange contribution is the kaon-exchange Fock term of Fig. 2 which gives a small repulsive contribution to the potential [26]. As explained in Ref. [26], being one pion exchange forbidden, the leading pieces contributing to the long range part of the potential will come from two pion exchange terms. In this work, we will consider the terms represented in Fig. 1. The nucleon lines represent in medium nucleon propagators

$$
G(p)=\frac{\theta\left(|\vec{p}|-k_{f}\right)}{\gamma \cdot p-m+i \epsilon}+\frac{\theta\left(k_{f}-|\vec{p}|\right)}{\gamma \cdot p-m-i \epsilon},
$$

where $k_{f}$ is the Fermi momentum. We start with diagram (a) of Fig. 1. Two pieces, direct and crossed, appear in the calculation of the $N h$ loop after doing the energy integration. In order to compare our results with Ref. [26], we calculate separately the part of the direct piece linear in the nucleon occupation number, $n(k)=\theta\left(k_{f}-|\vec{p}|\right)$. Furthermore, a nonrelativistic approximation is performed expanding the self-energy terms in a power series of an average baryon mass, $M_{B} \equiv\left(2 M_{N}+M_{\Lambda}+M_{\Sigma}\right) / 4$ and keeping only the leading order. We have checked numerically that this approximation is good and simplifies considerably the formulas. We also define the following variables related to mass splittings: $M_{\Sigma}-M_{\Lambda} \equiv$ $\Delta^{2} / M_{B}, M_{\Sigma^{*}}-M_{\Lambda} \equiv \Delta^{* 2} / M_{B}$, and $M_{\Delta}-M_{N} \equiv \Delta_{f}^{2} / M_{B}$, giving $\Delta=285 \mathrm{MeV}, \Delta_{f}=553 \mathrm{MeV}$, and $\Delta^{*}=532 \mathrm{MeV}$.

After integration over the energy variable in both loops, the direct term linear in $n$ of diagram (a) of Fig. 1 gives the

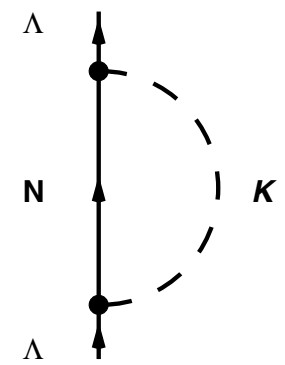

FIG. 2. One kaon exchange Fock diagram. following contribution to the mean-field:

$$
\begin{aligned}
U_{\Lambda}\left(k_{f}\right)^{N h-l}= & -\frac{D^{2} g_{A}^{2}}{f_{\pi}^{4}} \int_{|\vec{p}|<k_{f}} \frac{d^{3} p d^{3} l}{(2 \pi)^{6}} \\
& \times \frac{M_{B} \vec{l}^{4}}{\left(m_{\pi}^{2}+\vec{l}^{2}\right)^{2}\left(\Delta^{2}+\vec{l}^{2}-\vec{l} \cdot \vec{p}\right)},
\end{aligned}
$$

where $g_{A}=D+F, \vec{l}$ is the momentum of the pion in the two-pion exchange loop and $\vec{p}$ is the momentum of the nucleon in the pion self-energy loop. The denominator of the integrand shows clearly how the smallness of the mass of the pion and of the $\Delta$ splitting enhances the importance of low momenta $l$ as compared to the exchange of heavier mesons. This integral can be done analytically subtracting $\frac{M_{B}}{l^{2}}$ from the integrand [26]. Then, this divergent piece is regularized with a cutoff $\bar{\Lambda}$ and the remaining part is integrated from 0 to infinity. The result is

$$
\begin{aligned}
U_{\Lambda}\left(k_{f}\right)^{N h-l}= & D^{2} g_{A}^{2} \frac{M_{B}}{\left(2 \pi f_{\pi}\right)^{4}} \\
& \times\left\{-4 \frac{\bar{\Lambda}}{3} k_{f}^{3}+\pi m_{\pi}^{3} k_{f} \phi\left(\frac{k_{f}^{2}}{m_{\pi}^{2}}, \frac{\Delta^{2}}{m_{\pi}^{2}}\right)\right\},
\end{aligned}
$$

with [26]

$$
\begin{aligned}
\phi(x, y)= & y-3+\frac{1}{4}(x-2 y+6) \sqrt{4 y-x}+\frac{2}{\sqrt{x}} \\
& \times\left(2 x+y^{2}-4 y+3\right) \arctan \frac{\sqrt{x}}{2+\sqrt{4 y-x}} .
\end{aligned}
$$

The function $\phi\left(\frac{k_{f}^{2}}{m_{\pi}^{2}}, \frac{\Delta^{2}}{m_{\pi}^{2}}\right)$ depends only on the low mass scales $k_{f}$ and $\Delta$. One might interpret the $\bar{\Lambda}$ term as effectively parametrizing attractive contact pieces and the $\phi$ term as being a proper model independent long range part which only depends on physical quantities like masses and coupling constants. This long range part is repulsive, and only for big enough values of the cutoff $(\sim 0.5 \mathrm{GeV}) U_{\Lambda}\left(k_{f}\right)^{N h-l}$ becomes attractive.

At leading order in $M_{B}$, the contribution of all other parts of diagram (a) of Fig. 1 reduces to

$$
\begin{aligned}
U_{\Lambda}\left(k_{f}\right)^{N h-o}= & \frac{D^{2} g_{A}^{2}}{f_{\pi}^{4}} \int_{|\vec{p}|,|\vec{k}|<k_{f}} \frac{d^{3} p d^{3} k}{(2 \pi)^{6}} \\
& \times \frac{M_{B}(\vec{p}-\vec{k})^{4}}{\left[m_{\pi}^{2}+(\vec{p}-\vec{k})^{2}\right]^{2}\left[\Delta^{2}+\vec{k}^{2}-\vec{p} \cdot \vec{k}\right]},
\end{aligned}
$$

where we have introduced a suitable change of variables $\vec{p}+\vec{l}=\vec{k}$. This integral is convergent and can be evaluated numerically, producing a small repulsion, $U_{\Lambda}\left(k_{f 0}\right)^{N h-o}=$ 7.45 MeV at normal nuclear density.

The diagram (b) of Fig. 1 considers the excitation of a $\Delta$-hole instead of a nucleon-hole. After integration over the energy variables in both loops and taking the leading order in $M_{B}$ its contribution to the potential is given uniquely 
by

$$
\begin{aligned}
U_{\Lambda}\left(k_{f}\right)^{\Delta \mathrm{h}}= & -\frac{8 D^{2} \mathcal{C}_{\Delta}^{2}}{9 f_{\pi}^{4}} \int_{|\vec{p}|<k_{f}} \frac{d^{3} p d^{3} l}{(2 \pi)^{6}} \\
& \times \frac{M_{B} \vec{l}^{4}}{\left(m_{\pi}^{2}+\vec{l}^{2}\right)^{2}\left(\Delta^{2}+\Delta_{f}^{2}+\vec{l}^{2}-\vec{l} \cdot \vec{p}\right)} .
\end{aligned}
$$

The ratio $\frac{8 \mathcal{C}_{\Delta}^{2}}{9 g_{A}^{2}}=2.1$ shows the larger coupling of pions to $\Delta$ 's than to nucleons. This factor partly compensates the damping produced by the extra $\Delta_{f}^{2}$ term in the denominator.

The (c) and (d) diagrams consider the hyperon $\Sigma^{*}(1385)$ instead of $\Sigma$ as intermediate state. Their contribution is given by formulas with the same structure as the previous ones but changing of the mass splittings ( $\Delta \rightarrow \Delta^{*}$ ) and the coefficient in front of Eqs. (4), (7) and (8):

$$
\begin{aligned}
U_{\Lambda}^{*}\left(k_{f}\right)^{N h-l}= & -\frac{\mathcal{C}_{\Sigma *}^{2} g_{A}^{2}}{2 f_{\pi}^{4}} \int_{|\vec{p}|<k_{f}} \frac{d^{3} p d^{3} l}{(2 \pi)^{6}} \\
& \times \frac{M_{B} \vec{l}^{4}}{\left(m_{\pi}^{2}+\vec{l}^{2}\right)^{2}\left(\Delta^{* 2}+\vec{l}^{2}-\vec{l} \cdot \vec{p}\right)}, \\
U_{\Lambda}^{*}\left(k_{f}\right)^{N h-o}= & \frac{\mathcal{C}_{\Sigma *}^{2} g_{A}^{2}}{2 f_{\pi}^{4}} \int_{|\vec{p}|,|\vec{k}|<k_{f}} \frac{d^{3} p d^{3} k}{(2 \pi)^{6}} \\
& \times \frac{M_{B}(\vec{p}-\vec{k})^{4}}{\left[m_{\pi}^{2}+(\vec{p}-\vec{k})^{2}\right]^{2}\left[\Delta^{* 2}+\vec{k}^{2}-\vec{p} \cdot \vec{k}\right]}, \\
U_{\Lambda}^{*}\left(k_{f}\right)^{\Delta \mathrm{h}}= & -\frac{4 \mathcal{C}_{\Sigma *}^{2} \mathcal{C}_{\Delta}^{2}}{9 f_{\pi}^{4}} \int_{|\vec{p}|<k_{f}} \frac{d^{3} p d^{3} l}{(2 \pi)^{6}} \\
& \times \frac{M_{B} \vec{l}^{4}}{\left(m_{\pi}^{2}+\vec{l}^{2}\right)^{2}\left(\Delta^{* 2}+\Delta_{f}^{2}+\vec{l}^{2}-\vec{l} \cdot \vec{p}\right)} .
\end{aligned}
$$

Again, the integration in Eq. (10) does not require regularization and gives a quite small contribution, $U_{\Lambda}^{*}\left(k_{f 0}\right)^{N h-o}=$ $5.83 \mathrm{MeV}$ at $\rho=\rho_{0}$. On the other hand, it is obvious that the integrations of Eqs. (8), (9), and (11), which give the main contributions to the potential can be done analytically in the same manner as Eq. (4) after subtracting from the integrand the divergent piece $\frac{M_{B}}{l^{2}}$, later integrated with a cutoff. Their total contribution $U_{\Lambda}\left(k_{f}\right)^{N h-l}+U_{\Lambda}\left(k_{f}\right)^{\Delta \mathrm{h}}+U_{\Lambda}^{*}\left(k_{f}\right)^{N h-l}+$ $U_{\Lambda}^{*}\left(k_{f}\right)^{\Delta \mathrm{h}}$ is then

$$
\begin{aligned}
U_{\Lambda}\left(k_{f}\right)^{d}= & D^{2} g_{A}^{2} \frac{M_{B}}{\left(2 \pi f_{\pi}\right)^{4}}\left\{-4 \frac{\bar{\Lambda}^{\mathrm{eff}}}{3} k_{f}^{3}\right. \\
& +\pi m_{\pi}^{3} k_{f}\left(\phi\left(\frac{k_{f}^{2}}{m_{\pi}^{2}}, \frac{\Delta^{2}}{m_{\pi}^{2}}\right)+\frac{8 \mathcal{C}_{\Delta}^{2}}{9 g_{A}^{2}} \phi\right. \\
& \times\left(\frac{k_{f}^{2}}{m_{\pi}^{2}}, \frac{\Delta^{2}+\Delta_{f}^{2}}{m_{\pi}^{2}}\right)+\frac{\mathcal{C}_{\Sigma *}^{2}}{2 D^{2}} \phi\left(\frac{k_{f}^{2}}{m_{\pi}^{2}}, \frac{\Delta^{* 2}}{m_{\pi}^{2}}\right) \\
& \left.\left.+\frac{4 \mathcal{C}_{\Sigma *}^{2} \mathcal{C}_{\Delta}^{2}}{9 D^{2} g_{A}^{2}} \phi\left(\frac{k_{f}^{2}}{m_{\pi}^{2}}, \frac{\Delta^{* 2}+\Delta_{f}^{2}}{m_{\pi}^{2}}\right)\right)\right\},
\end{aligned}
$$

where, if the same cutoff is used for all integrations,

$$
\bar{\Lambda}^{\text {eff }}=\bar{\Lambda}\left(1+\frac{8 \mathcal{C}_{\Delta}^{2}}{9 g_{A}^{2}}+\frac{\mathcal{C}_{\Sigma *}^{2}}{2 D^{2}}+\frac{4 \mathcal{C}_{\Sigma *}^{2} \mathcal{C}_{\Delta}^{2}}{9 D^{2} g_{A}^{2}}\right) .
$$

The net effect of all $\phi$ pieces is a strong repulsion, that needs to be compensated by a strong short range attraction. This is effectively accomplished by the cut-off term which is proportional to the density and is equivalent to a contact term. In Ref. [26], it was suggested that the contributions of the new diagrams evaluated in this work and other short range pieces, at the densities of interest, could be accounted for by a term linear in density, $\left(\sim k_{f}^{3}\right)$. We have checked that this argument is qualitatively correct but some word of caution is needed. Indeed, performing a Taylor expansion of Eq. (12) and considering separately the different diagrams we find that the relative size of the higher order terms, like $k_{f}^{5}$, compared to the $k_{f}^{3}$ term is smaller for the heavier mass states because of the larger mass splittings. However, the new terms are multiplied by larger coupling constants, so that the net contribution of the nonlinear pieces of the new diagrams is comparable to the one obtained in Ref. [26].

In Fig. 3, we show the results for the different terms and the total for a cut-off $\bar{\Lambda}=1077 \mathrm{MeV}$, which has been adjusted to produce a potential of $-30 \mathrm{MeV}$ at $\rho=\rho_{0}$. We also include the contribution of the kaon Fock term (Fig. 2) taken from Ref. [26]. It is clear from the results that the new pieces originating from the coupling of the pions to the baryons decuplet have a large effect, although their interpretation in terms of separation of small and large energy scales is not so neat anymore as for the first diagram of Fig. 1 because of the larger mass splittings. Also, because of the regularization procedure the results depend linearly in the cutoff and their relative importance and/or size is rather arbitrary. Furthermore, in the figure for illustration purposes, we have assigned to each of the diagrams a part from the $\bar{\Lambda}^{\text {eff }}$ term proportional to its coupling constants [see Eq. (13)]. However, one should remember that the $\bar{\Lambda}^{\text {eff }}$ term has been fitted to adjust the value

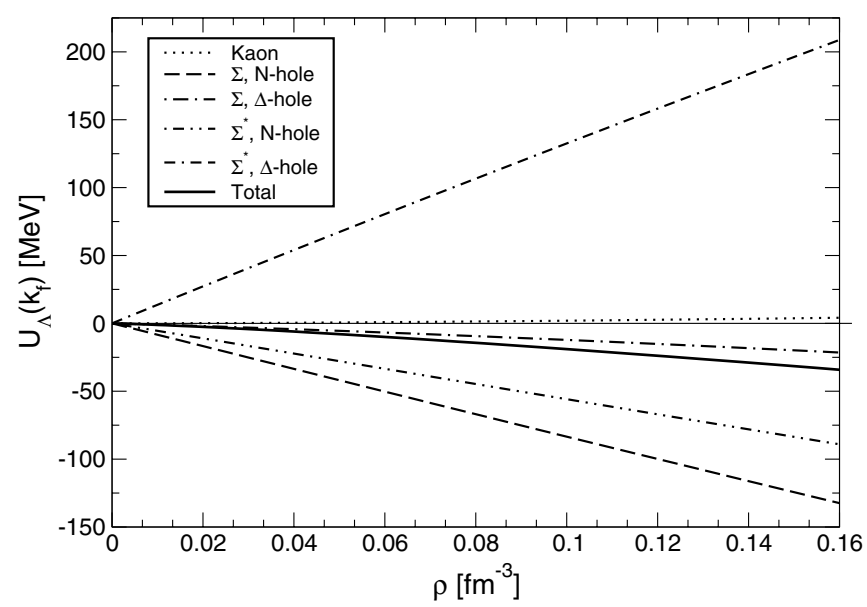

FIG. 3. $\Lambda$ mean field $U_{\Lambda}\left(k_{f}\right)$ dependence on density obtained using Eq. (12) for the direct terms. The cross terms are evaluated numerically. The kaon Fock contribution is taken from [26]. 


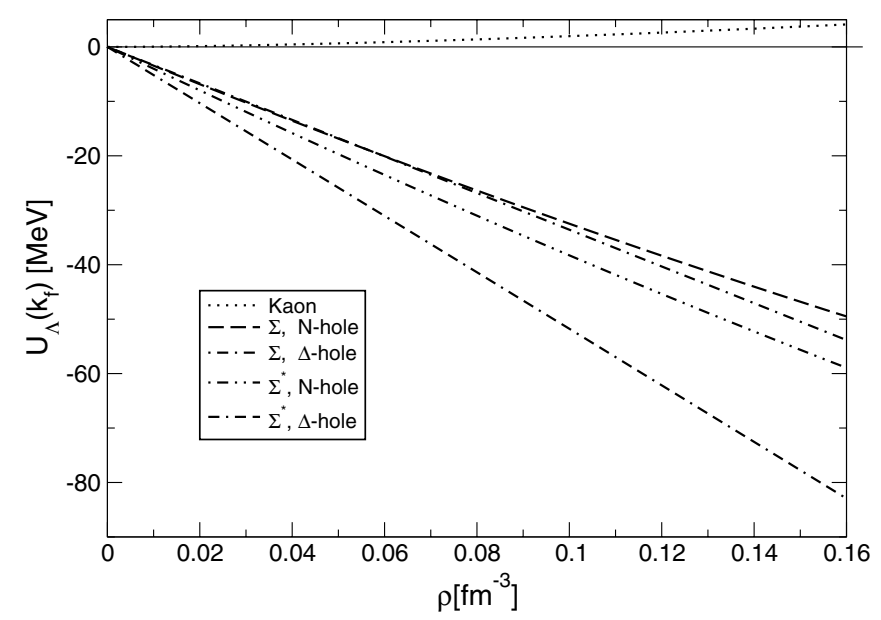

FIG. 4. $\Lambda$ mean field $U_{\Lambda}\left(k_{f}\right)$ dependence on density with a cut-off regularization, $\bar{\Lambda}=600 \mathrm{MeV}$.

of the potential at $\rho=\rho_{0}$ and thus it is assumed to also account for the contribution of short range pieces.

Alternatively, we could regularize all integrations directly with the use of a cutoff prior to any subtraction. This differs from the previous approach because the $l$ cutoff also affects the convergent pieces. Although the difference between both approaches is of order $O\left(\bar{\Lambda}^{-1}\right)$ it cannot be neglected except for values much larger than $1 \mathrm{GeV}$. On the other hand this procedure which cuts high momentum transfers is closer to the typical meson exchange potentials that incorporate form factors. In Fig. 4, we show the results for the different terms and the total for a cutoff $\bar{\Lambda}=600 \mathrm{MeV}$. We see that all terms are of a similar size. The reason is the large coupling of the baryon decuplet that partly cancels the effect of the larger masses in the denominator. Compare for instance diagrams (a) and (b) of Fig. 1. They are related to the pion self-energy coming from particle-hole and $\Delta$-hole excitations, respectively, and it is well known the importance of the $\Delta$-hole part even at very low energies. It is remarkable the large size of the contribution from diagram (d). In this case, all vertices correspond to octet-decuplet transitions which are very large. Obviously, the total potential is too large and shorter range pieces are required. (See, for instance, Ref. [37] where the inclusion of short range correlations in these pieces leads to reasonable total potentials.) Also noticeable are the large differences in both size and pattern of the individual contributions between Figs. 3 and 4, which come from the regularization procedures. We find that the two approaches converge for rather large values of the cutoff $(\Lambda \simeq 2.5 \mathrm{GeV})$.

\section{III. $\Lambda$-NUCLEUS SPIN-ORBIT POTENTIAL}

The empirical result that the $\Lambda$-nucleus spin-orbit coupling is very small compared with those corresponding to nucleons in ordinary nuclei presents an intriguing problem in lowenergy hadron physics. As discussed in the Introduction, many attempts have tried to explain this fact. As an example, in scalar-vector relativistic mean-field models [22] a large tensorial $\omega$ - $\Lambda$ coupling of opposite sign to the vector coupling accounts for the cancellation of the large spin-orbit potential produced by the vector coupling of the $\omega$ meson. In Ref. [26], it was found that the first of the two pion exchange terms represented in Fig. 1 gives a quite natural explanation of this puzzle as these terms produce a spin-orbit potential of opposite sign and of similar magnitude to that produced by the vector coupling of the $\omega$ meson. And more importantly, they do so in a model independent way, as their size and sign depend only on known couplings and masses and no regularization is required. In this section we will study if that result is still valid once the other processes shown in Fig. 1 are included.

The spin-orbit coupling is obtained from the spin-dependent part of the self-energy produced when we consider the interaction of the corresponding particle (in our case a $\Lambda$-hyperon) with a weakly inhomogeneous medium. As explained in Refs. $[26,38]$, the spin-orbit part of the optical potential is calculated by considering that the $\Lambda$-hyperon scatters from an initial three-momentum $\vec{p}_{a}-\vec{q} / 2$ to a final three-momentum $\vec{p}_{a}+$ $\vec{q} / 2$. Then, the spin-orbit part for such weak inhomogeneity arises as

$$
\Sigma_{\text {spin }}=\frac{i}{2} \vec{\sigma} \cdot\left(\vec{q} \times \vec{p}_{a}\right) U_{\Lambda l s}\left(k_{f}\right),
$$

where the spin-orbit strength $U_{\Lambda l s}\left(k_{f}\right)$ is taken in the limit $\vec{q}=\vec{p}_{a}=0$, and for a homogeneous medium of Fermi momentum $k_{f}$. In detail, this structure is obtained manipulating the expression $\vec{\sigma} \cdot(\vec{l}-\vec{q} / 2) \vec{\sigma} \cdot(\vec{l}+\vec{q} / 2)$ coming from the $\pi \Sigma \Lambda$ vertex in the two first diagrams of Fig. 1, and $\vec{S} \cdot(\vec{l}-\vec{q} / 2) \vec{S}^{\dagger} \cdot(\vec{l}+\vec{q} / 2)$ coming from the $\pi \Sigma^{*} \Lambda$ vertex of the two last diagrams of Fig. 1. Using the known relations

$$
\sigma_{i} \sigma_{j}=\delta_{i j}+i \epsilon_{i j k} \sigma_{k}
$$

and

$$
S_{i} S_{j}^{\dagger}=2 / 3 \delta_{i j}-i / 3 \epsilon_{i j k} \sigma_{k}
$$

we obtain the antisymmetric tensorial structure which characterizes this term of the self-energy [Eq. (14)]. Notice that these pieces have different sign attending to the SU(3)-multiplet which the internal-line baryon belongs to, circumstance that will produce cancellations between the diagrams with $\Sigma$ and the diagrams with $\Sigma^{*}$. The other factor, $\vec{p}_{a}$ comes from the denominator in the integrand and arises after expanding the amplitude in a power series and keeping only the linear term. Finally, using $\int d \Omega l^{i} l^{j}=l^{2} / 3 \delta_{i j}\left(\int d \Omega\right)$, we obtain the following $\Lambda$-nucleus spin-orbit potentials for the different diagrams considered:

$$
\begin{aligned}
U_{\Lambda l s}\left(k_{f}\right)^{N h-l}= & -\frac{2 D^{2} g_{A}^{2}}{3 f_{\pi}^{4}} \int_{|\vec{p}|<k_{f}} \frac{d^{3} p d^{3} l}{(2 \pi)^{6}} \\
& \times \frac{M_{B} \vec{l}^{4}}{\left(m_{\pi}^{2}+\vec{l}^{2}\right)^{2}\left(\Delta^{2}+\vec{l}^{2}-\vec{l} \cdot \vec{p}\right)^{2}},
\end{aligned}
$$




$$
\begin{aligned}
& U_{\Lambda l s}\left(k_{f}\right)^{N h-o}=\frac{2 D^{2} g_{A}^{2}}{3 f_{\pi}^{4}} \int_{|\vec{p}|,|\vec{k}|<k_{f}} \frac{d^{3} p d^{3} k}{(2 \pi)^{6}} \\
& \times \frac{M_{B}(\vec{p}-\vec{k})^{4}}{\left[m_{\pi}^{2}+(\vec{p}-\vec{k})^{2}\right]^{2}\left[\Delta^{2}+\vec{k}^{2}-\vec{p} \cdot \vec{k}\right]^{2}}, \\
& U_{\Lambda l s}\left(k_{f}\right)^{\Delta \mathrm{h}}=-\frac{16 D^{2} \mathcal{C}_{\Delta}^{2}}{27 f_{\pi}^{4}} \int_{|\vec{p}|<k_{f}} \frac{d^{3} p d^{3} l}{(2 \pi)^{6}} \\
& \times \frac{M_{B} \vec{l}^{4}}{\left(m_{\pi}^{2}+\vec{l}^{2}\right)^{2}\left(\Delta^{2}+\Delta_{f}^{2}+\vec{l}^{2}-\vec{l} \cdot \vec{p}\right)^{2}}, \\
& U_{\Lambda l s}^{*}\left(k_{f}\right)^{N h-l}=\frac{\mathcal{C}_{\Sigma *}^{2} g_{A}^{2}}{6 f_{\pi}^{4}} \int_{|\vec{p}|<k_{f}} \frac{d^{3} p d^{3} l}{(2 \pi)^{6}} \\
& \times \frac{M_{B} \vec{l}^{4}}{\left(m_{\pi}^{2}+\vec{l}^{2}\right)^{2}\left(\Delta^{* 2}+\vec{l}^{2}-\vec{l} \cdot \vec{p}\right)^{2}}, \\
& U_{\Lambda l s}^{*}\left(k_{f}\right)^{N h-o}=-\frac{\mathcal{C}_{\Sigma *}^{2} g_{A}^{2}}{6 f_{\pi}^{4}} \int_{|\vec{p}|,|\vec{k}|<k_{f}} \frac{d^{3} p d^{3} k}{(2 \pi)^{6}} \\
& \times \frac{M_{B}(\vec{p}-\vec{k})^{4}}{\left[m_{\pi}^{2}+(\vec{p}-\vec{k})^{2}\right]^{2}\left[\Delta^{* 2}+\vec{k}^{2}-\vec{p} \cdot \vec{k}\right]^{2}}, \\
& U_{\Lambda l s}^{*}\left(k_{f}\right)^{\Delta \mathrm{h}}=\frac{4 \mathcal{C}_{\Sigma *}^{2} \mathcal{C}_{\Delta}^{2}}{27 f_{\pi}^{4}} \int_{|\vec{p}|<k_{f}} \frac{d^{3} p d^{3} l}{(2 \pi)^{6}} \\
& \times \frac{M_{B} \vec{l}^{4}}{\left(m_{\pi}^{2}+\vec{l}^{2}\right)^{2}\left(\Delta^{* 2}+\Delta_{f}^{2}+\vec{l}^{2}-\vec{l} \cdot \vec{p}\right)^{2}} .
\end{aligned}
$$

All these integrations are convergent and therefore do not depend in other input parameters than the coupling constants and particle masses. Notice also that they are not a relativistic correction since they arise at leading order in a $M_{B}$ expansion, the same order as the central potential discussed before. This is a different situation to that which emerges in mean-field models with OBE interactions, where the spin-orbit interaction appears as a higher order correction [39]. We have checked numerically that the expansion in $M_{B}$ is quite good, even when the mass splittings are almost $300 \mathrm{MeV}$. The difference at $\rho=\rho_{0}$ is less than $10 \%$ for all diagrams.

In Fig. 5, it is shown the density dependence of the spinorbit potentials calculated in this manner. The $\Delta$-hole diagram (b) gives a contribution similar in size and of the same sign as the $N$-hole diagram (a). This would spoil the result of Ref. [26] and produce a too large negative contribution. However, the processes with a $\Sigma^{*}$ have a positive contribution giving a total result quite similar to that obtained previously including only the diagram (a). As explained before, this different sign comes from the opposite sign of the antisymmetric parts of Eqs. (15) and (16), which correspond to octet-octet and octet-decuplet spin transition operators, respectively.

We also show in Fig. 5 a rough estimate of the total result by using the same approach as in Ref. [26] to account for the missing short range pieces. A full discussion justifying this

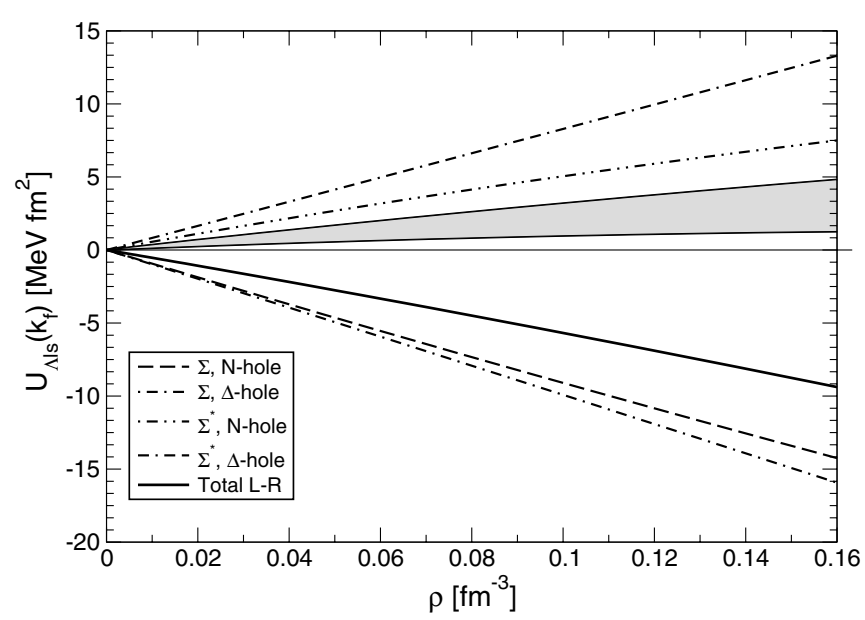

FIG. 5. Spin-orbit potential $U_{\Lambda l s}\left(k_{f}\right)$ of a $\Lambda$-hyperon in isospinsymmetric nuclear matter for the diagrams of Fig. 2 (solid line). The shadowed band shows the total SO potential after adding the short range part parametrized as described in the text for $C_{l}$ values between $1 / 2$ and $2 / 3$ and $U_{\Lambda l s}^{\mathrm{sr}}\left(k_{f}\right)=21.3 C_{l} \mathrm{MeV} \mathrm{fm}^{2} \rho / \rho_{0}$.

approach can be found there. We take

$$
U_{\Lambda l s}^{\text {shell }}\left(k_{f}\right)=C_{l} \frac{M_{N}^{2}}{M_{\Lambda}^{2}} U_{N l s}^{\text {shell }}\left(k_{f}\right),
$$

where the factor $M_{N}^{2} / M_{\Lambda}^{2}$ comes from the replacement of the nucleon by the $\Lambda$-hyperon in these relativistic spin-orbit terms. For $U_{N l s}^{\text {shell }}\left(k_{f}\right)$ we suppose a linear dependence in $\rho$ that takes the value $30 \mathrm{MeV} \mathrm{fm}^{3}$ at saturation density [40]. For $C_{l}$ we take the band between the values $1 / 2$ and $2 / 3$. We find that the sum of long range pieces, after inclusion of the decuplet baryons, still produces a negative spin-orbit contribution of a similar magnitude to the short range pieces, leading to a final estimation of a small value of the spin-orbit potential.

\section{SUMMARY}

We have studied the long range part of the $\Lambda$-hyperon optical potential in nuclei using flavor-SU(3) chiral Lagrangians. In a previous work [26], the kaon Fock exchange term and the two pion exchange term with the excitation of a nucleon-hole have been studied. We have extended that work adding the contributions of other two-pion exchange pieces that appear when the baryons decuplet is considered. The inclusion of the new processes with the $\Delta(1232)$ and $\Sigma(1385)$ resonances has been shown to be quite relevant, even when their masses are relatively large, due to their strong couplings to the pions and the baryons octet.

The central part of the potential can be fitted to the empirical result by choosing appropriately the cutoff used in the regularization. Therefore, although some interesting separation of low and high mass scales can be done, we do not have any real predictability here for the full size of the potential. Using the analytical procedure of regularization with a cutoff $\bar{\Lambda}=1.077 \mathrm{GeV}$ we get the typical $30 \mathrm{MeV}$ attraction. This value of the cutoff should be interpreted with care as it is not as much a limit for momenta as a parametrization for 
short-range pieces. With these caveats in mind, we find that the contributions of the new diagrams have the same analytical structure and are of similar or larger size as the previously studied excitation of a nucleon-hole.

We also consider the contribution to the spin-orbit potential that comes out from these terms. This contribution is model independent, as it does not require any regularization and depends only on physical parameters like masses and coupling constants. Our results support the explanation of the smallness of the $\Lambda$-nuclear spin-orbit interaction due to cancellation between short and long range pieces and shows the importance of the $\Sigma^{*}$ and $\Delta$ degrees of freedom for the hyperon-nucleus interactions. The different sign in the sum over spins of the internal baryon lines for $\Sigma$ and $\Sigma^{*}$ is crucial for this result.

\section{ACKNOWLEDGMENTS}

This work was partially supported by DGI and FEDER funds, contract no. BFM2003-00856 and by the EU Integrated Infrastructure Initiative Hadron Physics Project contract no. RII3-CT-2004-506078. J.M.C. acknowledges support from CSIC-Fundación Bancaja. The authors want to thank M. Döring for useful discussions.
[1] B. Povh, Annu. Rev. Nucl. Part. Sci. 28, 1 (1978).

[2] C. B. Dover and A. Gal, Prog. Part. Nucl. Phys. 12, 171 (1985).

[3] D. J. Millener, C. B. Dover, and A. Gal, Phys. Rev. C 38, 2700 (1988).

[4] C. B. Dover, D. J. Millener, and A. Gal, Phys. Rep. 184, 1 (1989).

[5] E. Oset, P. Fernandez de Cordoba, L. Salcedo, and R. Brockmann, Phys. Rep. 188, 79 (1990).

[6] R. E. Chrien and C. B. Dover, Annu. Rev. Nucl. Part. Sci. 39, 113 (1989).

[7] H. Bando, T. Motoba, and J. Zofka, Int. J. Mod. Phys. A 5, 4021 (1990).

[8] B. F. Gibson and E. V. Hungerford, Phys. Rep. 257, 349 (1995).

[9] I. Vidana, A. Polls, A. Ramos, and M. Hjorth-Jensen, Nucl. Phys. A644, 201 (1998).

[10] E. Hiyama, M. Kamimura, T. Motoba, T. Yamada, and Y. Yamamoto, Phys. Rev. Lett. 85, 270 (2000).

[11] I. Vidana, A. Polls, A. Ramos, and H. J. Schulze, Phys. Rev. C 64, 044301 (2001).

[12] B. Holzenkamp, K. Holinde, and J. Speth, Nucl. Phys. A500, 485 (1989).

[13] T. A. Rijken, V. G. J. Stoks, and Y. Yamamoto, Phys. Rev. C 59, 21 (1999).

[14] J. Haidenbauer and U. G. Meissner, Phys. Rev. C 72, 044005 (2005).

[15] A. Bouyssy, Nucl. Phys. A290, 324 (1977).

[16] R. Brockmann and W. Weise, Phys. Lett. B69, 167 (1977).

[17] W. Bruckner et al. (Heidelberg-Saclay-Strasbourg Collaboration), Phys. Lett. B79, 157 (1978).

[18] M. May et al., Phys. Rev. Lett. 47, 1106 (1981).

[19] M. May et al., Phys. Rev. Lett. 51, 2085 (1983).

[20] S. Ajimura et al., Phys. Rev. Lett. 86, 4255 (2001).
[21] J. V. Noble, Phys. Lett. B89, 325 (1980).

[22] B. K. Jennings, Phys. Lett. B246, 325 (1990).

[23] H. J. Pirner, Phys. Lett. B85, 190 (1979).

[24] K. Tsushima, K. Saito, J. Haidenbauer, and A. W. Thomas, Nucl. Phys. A630, 691 (1998).

[25] Y. Fujiwara, M. Kohno, T. Fujita, C. Nakamoto, and Y. Suzuki, Nucl. Phys. A674, 493 (2000).

[26] N. Kaiser and W. Weise, Phys. Rev. C 71, 015203 (2005).

[27] N. Kaiser, Phys. Rev. C 71, 068201 (2005).

[28] N. Kaiser, S. Fritsch, and W. Weise, Nucl. Phys. A697, 255 (2002).

[29] N. Kaiser, S. Fritsch, and W. Weise, Nucl. Phys. A700, 343 (2002).

[30] S. Fritsch, N. Kaiser, and W. Weise, Nucl. Phys. A750, 259 (2005).

[31] E. Oset, H. Toki, and W. Weise, Phys. Rep. 83, 281 (1982).

[32] K. Sasaki, E. Oset, and M. J. Vicente Vacas, Phys. Rev. C 74, 064002 (2006).

[33] E. Oset and A. Ramos, Nucl. Phys. A635, 99 (1998).

[34] O. Dumbrajs, R. Koch, H. Pilkuhn, G. C. Oades, H. Behrens, J. J. De Swart, and P. Kroll, Nucl. Phys. B216, 277 (1983).

[35] M. N. Butler, M. J. Savage, and R. P. Springer, Nucl. Phys. B399, 69 (1993).

[36] M. Doring, E. Oset, and S. Sarkar, Phys. Rev. C 74, 065204 (2006).

[37] L. Tolos, A. Ramos, and E. Oset, Phys. Rev. C 74, 015203 (2006).

[38] N. Kaiser, Nucl. Phys. A709, 251 (2002).

[39] R. Brockmann and W. Weise, Phys. Rev. C 16, 1282 (1977).

[40] E. Chabanat, P. Bonche, P. Haensel, J. Meyer, and R. Schaeffer, Nucl. Phys. A635, 231 (1998). 